\title{
Vitamin A, carotenoid and vitamin E plasma concentrations in children from Laos in relation to sex and growth failure Florian J Schweigert*1, Jeannine Klingner ${ }^{1}$, Andrea Hurtienne ${ }^{1}$ and Hans J Zunft ${ }^{2}$
}

\begin{abstract}
Address: ${ }^{1}$ Department of Physiology and Pathophysiology, Institute of Nutritional Science, University of Potsdam, Germany and ${ }^{2}$ Department of Intervention Studies, German Institut of Nutritional Research, Germany, both Arthur-Scheunert-Allee 114-116, D-14558 Potsdam-Rehbrücke

Email: Florian J Schweigert* - fjschwei@rz.uni-potsdam.de; Jeannine Klingner - jeannine.klingner@gmx.de; Andrea Hurtienne - ahurti@rz.unipotsdam.de; Hans J Zunft - zunft@mail.dife.de

* Corresponding author
\end{abstract}

This article is available from: http://www.nutritionj.com/content/2/1/17

(c) 2003 Schweigert et al; licensee BioMed Central Ltd. This is an Open Access article: verbatim copying and redistribution of this article are permitted in all media for any purpose, provided this notice is preserved along with the article's original URL.

\begin{abstract}
Background: Deficiencies of vitamin A and its precursors, the carotenoids are common problems in developing countries. Plasma levels of these components are used as biomarkers of their availability. The study was conducted to evaluate whether blood plasma obtained from capillaries can be compared with plasma obtained from venous blood with regard to its levels of retinol, carotenoids and $\alpha$-tocopherol and secondly to apply this technique to evaluate the levels of these components in children in a region with possible deficiencies.
\end{abstract}

Methods: The survey was conducted in a region of Laos in 81 children (age 35 to 59 months). Dietary intake was assessed by a questionnaire. Retinol, carotenoids and $\alpha$-tocopherol were determined by HPLC. Blood plasma was obtained either from capillary blood collected into microcapillaries and for reasons of methodological comparison in 14 adults from venous blood.

Results: The comparison between capillary and venous blood revealed that all components except zeaxanthin were $9-23 \%$ higher in plasma obtained from capillary blood. Results in Laotian children showed that all investigated components except retinol were significantly lower $(P<0.0 I)$ compared to European children of slightly older age. Contrary to children in Europe, most components were significantly lower in boys compared to girls. In children from Laos, lutein was the dominant carotenoid, while in children in Europe, $\beta$-carotene was dominant. Within the Laotian children only a few differences were observed between stunted and non-stunted children and between children from lowland areas and high land areas.

Conclusions: Results show that in consideration of slightly lower levels than in venous blood, capillary blood can be used to evaluate retinol, carotenoids and $\alpha$-tocopherol as biomarkers of intake or status and to evaluate the possible effect of diet on absolute and relative carotenoid composition in children from Europe and Laos. Observed sex related differences might not be related to diet and would need further investigation. 


\section{Background}

On a worldwide scale, more than $1 / 3^{\text {rd }}$ of all children suffer from protein malnutrition or undernutrition, with the highest prevalence showing in the developing countries of Asia, Africa, Latin America and Oceania. In Asia 80\%, in Africa $15 \%$ and in Latin America $5 \%$ of all children are suffering from consequences caused by protein malnutrition or undernutrition [1]. For more than 230 million children an inadequate vitamin A supply is shown, with 13 million of them being affected by night blindness. The main cause for the loss of their eye sight of currently world wide more than 2.8 million preschool children is dietary vitamin A deficiency [2]. Moreover, insufficient intake of vitamins and minerals is suggested to be a cause for the high prevalence of several sub-clinical diseases and growth retardation. This is especially true for Laos, where UNICEF noticed a growth retardation in all Laotian preschool children, which was classified by the WHO to be the highest in the world. Concerning the widespread night blindness, the national vitamin A study established that the intake of mainly rice as dominant staple food together with recurrent infectious diseases are the main causes of the vitamin A deficiency [3-5].

Vitamin A deficiency is caused by either a deficiency of vitamin A itself or by a reduced availability of precursors of vitamin A, the carotenoids. The reduced availability is either caused by its limited presence in food or by a reduced absorption due to a reduced fat content of the diet or a reduced absorptive capacity of the gut [6]. In addition to their function as precursor of vitamin A [7], carotenoids together with vitamin $\mathrm{E}$ are important micronutrients to maintain health by protecting the body against free radical damage [8]. In this context different epidemiological studies show an association with a variety of degenerative diseases such as cancer, heart disease and age related macula degeneration [9].

Because carotenoids, tocopherol and retinol plasma levels are biomarkers of their uptake or status $[10,11]$ these components are frequently investigated in populations with an affluent lifestyle especially with regard to factors affecting plasma levels of these components [12]. Only a few studies have addressed this question in developing countries especially with regard to infants. This is, however, of specific importance to understand firstly how the plasma levels of these components are influenced and secondly how these components might determine longevity and long-term health.

Major reasons for the limited number of studies available on the plasma concentrations in individuals from developing countries are on the one hand logistical problems and on the other hand the limited individual and public acceptance of such kind of investigations. Logistical prob- lems include blood collection, serum preparation by centrifugation, transportation as well as short-term and longterm storage under cool or frozen conditions. Mothers are not very enthusiastic with regard to an invasive sampling of blood especially in their infants. Therefore methods that will avoid the invasive venous blood sampling and a majority of logistical problems as well would be of advantage to encourage more studies on the levels of vitamin A, $\alpha$-tocopherol and carotenoids in populations of developing countries.

The purpose of this study was thus to answer two questions. Firstly, whether plasma levels of retinol, $\alpha$-tocopherol and carotenoids determined in capillary blood will validly represent the respective venous blood levels and secondly, whether a method using capillary blood plasma is suitable to asses the possible relation of vitamin A, carotenoid and $\alpha$-tocopherol status in infants with sex, growth failure and living conditions in children from the high- and lowland region of the Bolikhamxay province Laos.

\section{Subjects and Methods Subjects}

Within a nutritional study of the Family Health Project in Laos in 2000/2001, a total 1514 Laotian preschool children of the Province of Bolikhamxay were examined with regard to their nutrition, health and socio-economic situation in order to determine different causal factors between infants having a stunted growth and those with a normal growth. All children aged 0 to 59 months were eligible for entry in this study. The regional ethic committee approved the study. Participation was voluntary and informed consent was obtained from all mothers of participating children.

\section{Data and sample collection}

Laotian field workers collected information by questionnaire that included social-economic-education status, nutritional habits and queries to diseases.

Anthropometric measurements comprising height and weight, were measured by standardized equipment.

The daily food intake by quantity and amount was investigated over three consecutive using an estimated food record. The results were analysed with a computer programme called INMUCAL, which were developed by the Institute of Nutrition of the Mahidol University in Bangkok. The food frequency questionnaire (FFQ) determined the regular nutrition habits of the sample children over a period of time. Afterwards the daily consumption were calculated. The mothers were asked in an interview to estimate how often (daily, weekly, monthly, yearly) the child eats given food items from a list. The amount of a 
consumed food item the mother had to specify by using the cup and spoon set from Thailand. The food item from the given list were scaled with a digital scale according to the different sizes of the cups and spoon (SOEHNLE attache 8020, max. $2 \mathrm{~kg}$, division $2 \mathrm{~g}$, Germany).

Blood samples were obtained from a total of 81 children of different sex aged between 35 and 59 months. Data for the European control group $(\mathrm{n}=101)$ has been published previously [13] and are included in this paper for reasons of comparison. Capillary blood was drawn into heparinized microcapillaries $(60 \mu \mathrm{l})$ by puncturing the pad of the finger. To obtain plasma samples the microcapillaries were immediately centrifuged at the site for $4 \mathrm{~min}$ utes at $11500 \mathrm{~g}$ in a battery-driven miniature centrifuge (M 1101, Bayer Diagnostik GmbH, Germany). The plasma was transferred into opaque Eppendorf cups and stored in an ice-filled box. Within four hours they were transferred to the nearest hospital to be frozen at $-15^{\circ} \mathrm{C}$. From there they were shipped by air on dry ice to Germany where they were stored at $-80^{\circ} \mathrm{C}$. Analysis was performed within six months.

In order to compare the carotenoid and vitamin concentrations between capillary and venous blood, blood samples were obtained simultaneously from 14 individuals of different sex and age recruited from the institutional staff who gave informal consent to this sampling. Venous blood samples were taken from the antibrachial vein, capillary blood was drawn into heparinized microcapillaries $(60 \mu \mathrm{l})$ by puncturing the pad of the finger. Samples were centrifuged and treated in a comparable way.

\section{Determination of retinol, carotenoids and $\alpha$-tocopherol by HPLC}

Carotenoids (lutein, zeaxanthin, $\alpha$-carotene, $\beta$-carotene, $\beta$-cryptoxanthin, lycopene), $\alpha$-tocopherol and retinol and retinyl esters were separated and quantified by reversedphased HPLC [14]. Due to the limited amount of sample available the method was modified. Briefly, $200 \mu \mathrm{l}$ of ethanol were added to $20 \mu \mathrm{l}$ plasma diluted with $180 \mu \mathrm{l} \mathrm{H}_{2} \mathrm{O}$. After vortexing for $30 \mathrm{sec}$, the samples were extracted twice with $\mathrm{n}$-hexane $(1 \mathrm{ml}$ each time stabilized with $0.05 \%$ butylated hydroxytoluene (BHT)) and vortexed for $3 \mathrm{~min}$. The supernatants were removed, pooled, and evaporated under nitrogen and reconstituted in $50 \mu \mathrm{l}$ isopropanol and injected $(40 \mu \mathrm{l})$ into the HPLC-system (Waters, Eschborn, Germany). Accuracy and precision of the analyses were verified using a standard reference material (SMR 968a fat-soluble vitamins in human serum; National Institute of Standards and Technology (NIST), Gaithersburg, MD, USA). Coefficient of variability over time using control plasma was less than $4 \%$ for carotenoids, retinol, retinyl esters and $\alpha$-tocopherol. The recovery rate was above $95 \%$ for all components.

\section{Statistical analyses}

Normally distributed data are expressed as means and standard deviation (SD), and non-normally distributed data are expressed as medians and ranges. Statistical analysis was performed with a paired t test. Probability values below 0.05 were considered significant.

\section{Results and Discussion}

Only a few studies have addressed the question of plasma carotenoid levels in children. These data however are important because nutritional deficiency has many detrimental effects with short and long-term consequences for children. These include growth retardation, reduced cognitive functions and vision as well as an increased incidence of infectious diseases. As single causes or in combination these factors increase the mortality rates in developing countries $[15,16]$. Therefore, monitoring the nutritional status is very important. Methods that facilitate the acquisition of such data are highly necessary because limited information still exists with regard to major determining influences on the levels of carotenoids, $\alpha$-tocopherol and retinol for children in developing countries. This also concerns the interaction with factors of nutritional origin, or the important influence of the health status such as the acute phase response during infections $[17,18]$. One major advantage would be gained by reducing the invasiveness of blood sampling and the reduction of sample volume necessary to analyse key nutrients such as retinol, carotenoids and $\alpha$-tocopherol $[19,20]$.

\section{Comparison of plasma composition between samples obtained from venous or capillary blood}

Table 1 shows differences in plasma retinol, individual carotenoids (lutein, zeaxanthin, $\alpha$-carotene, $\beta$-carotene, $\beta$ cryptoxanthin, lycopene) and $\alpha$-tocopherol between plasma obtained from the same individuals either by puncturing of a vein or the capillaries of the fingertip. In general, all components were higher in plasma obtained from capillary blood. The most obvious differences were observed for $\alpha$ - and $\beta$-carotene. This observation is in accordance with a previous study in which, however, only $\beta$-carotene and $\alpha$-tocopherol were investigated [21]. The increased levels have been attributed to the reduced fluid in capillaries due to differences in hydrostatic pressure and colloid osmotic pressure in the capillaries. In this case, however, one would expect that all components measured would show similar quantitative changes. Nevertheless, the differences between plasma obtained from capillaries or veins are in the same order of magnitude. Thus, capillary blood sampling is a valid procedure to determine micronutrients such as retinol, $\alpha$-tocopherol and carotenoids under field condition not only in developing countries but also in neonatal and pediatric medicine. With regard to the situation in developing countries, 
Table I: Differences in the concentration of retinol, carotenoids and $\alpha$-tocopherol $(\mu \mathrm{mol} / \mathrm{l} ;$ mean \pm SD) between plasma samples $(\mathrm{n}=$ 14) obtained from paired venous or capillary blood

\begin{tabular}{lcc}
\hline & Venous & capillary \\
\hline$\alpha$-Tocopherol & $20.80 \pm 10.02$ & $23.35 \pm 10.02^{*}$ \\
Retinol & $1.42 \pm 0.31$ & $1.69 \pm 0.39 *$ \\
Lutein & $0.21 \pm 0.08$ & $0.23 \pm 0.09$ \\
Zeaxanthin & $0.02 \pm 0.01$ & $0.02 \pm 0.01$ \\
$\beta$-Cryptoxanthin & $0.11 \pm 0.05$ & $0.13 \pm 0.06$ \\
$\alpha$-Carotene & $0.11 \pm 0.07$ & $0.14 \pm 0.090^{*}$ \\
$\beta$-Carotene & $0.54 \pm 0.28$ & $0.66 \pm 0.32^{*}$ \\
Lycopene & $0.19 \pm 0.12$ & $0.25 \pm 0.13^{* * *}$
\end{tabular}

$* \mathrm{P}<0.05 * * * \mathrm{P}<0.001$

Table 2: Differences in the concentration of retinol, carotenoids and $\alpha$-tocopherol ( $\mu \mathrm{mol} / \mathrm{l} ;$ mean \pm SD) between European and Laotian children and effect of sex

\begin{tabular}{|c|c|c|c|c|}
\hline & \multicolumn{2}{|c|}{ Europe } & \multicolumn{2}{|c|}{ Laos } \\
\hline & all sexes & all sexes & girls & boys \\
\hline Girls / Boys & 101 & 81 & 45 & 36 \\
\hline$\alpha$-Tocopherol & $15.84 \pm 4.94$ & $1.885 \pm 1.755$ & $3.380 \pm 1.805$ & $0.469 \pm 1.504^{*}$ \\
\hline Retinol & $1.22 \pm 0.27$ & $0.967 \pm 0.230$ & $1.048 \pm 0.247$ & $0.931 \pm 0.178^{*}$ \\
\hline Lutein & $0.31 \pm 0.21$ & $0.063 \pm 0.050$ & $0.062 \pm 0.052$ & $0.038 \pm 0.038^{* * *}$ \\
\hline Zeaxanthin & $0.02 \pm 0.02$ & $0.005 \pm 0.004$ & $0.004 \pm 0.005$ & $0.003 \pm 0.002^{* * *}$ \\
\hline$\beta$-Cryptoxanthin & $0.16 \pm 0.14$ & $0.015 \pm 0.017$ & $0.009 \pm 0.020$ & $0.005 \pm 0.011^{* * * *}$ \\
\hline$\alpha$-Carotene & $0.65 \pm 0.43$ & $0.005 \pm 0.010$ & $0.003 \pm 0.003$ & $0.002 \pm 0.007$ \\
\hline$\beta$-Carotene & $1.57 \pm 1.15$ & $0.017 \pm 0.020$ & $0.014 \pm 0.018$ & $0.006 \pm 0.018^{* * *}$ \\
\hline Lycopene & $0.26 \pm 0.10$ & Traces & traces & traces \\
\hline
\end{tabular}

$* \mathrm{P}<0.05 * * * \mathrm{P}<0.001$

the procedure not only eliminates invasive blood sampling but also greatly reduces the equipment needed. Plasma can be obtained using a battery powered centrifuge and the small sample volumes can be stored and transported in well isolated storage containers even under less favourable conditions.

\section{Concentration of retinol, carotenoids and $\alpha$-tocopherol in plasma samples of children from Laos}

Table 2 summarizes the results on plasma concentrations of retinol, carotenoids and $\alpha$-tocopherol and shows obvious differences between groups of children sampled in Europe and in Laos as well as sex differences in Laotian children. Studies that determined the levels of these components in plasma are limited and mostly restricted to developed countries. One reason for this, as has been pointed out earlier, are the difficulties to obtain appropriate samples. As reported for adults and children in Nepal and Africa the levels observed for carotenoids are substantially lower in children from Laos compared to slightly older children from Austria [13] or Germany [22] or from much older ones in the USA [23]. Similarly low levels were found in children from Nigeria and Pakistan [24,25]. The differences in dietary carotenoid pattern are further strengthened by the striking differences in the carotenoid pattern. While in European infants the dominant carotenoid was $\beta$-carotene representing $52 \%$ of total carotenoids, in infants from Laos the dominant carotenoid was lutein with $62 \%(\mathrm{P}<0.001)$ (Table 3$)$. Both lower carotenoid plasma levels as well as differences in the plasma carotenoid pattern clearly reflects differences in the quantitiative and qualitative availability of carotenoids. High lutein levels are indicative of a consumption of dark green leafy vegetable and are frequently found as dominant carotenoid in plasma samples obtained from populations in developing countries [24]. The relative contribution of individual carotenoids to total carotenoids in Laotian children was independent of origin or sex (data not presented).

The numerically smaller difference between the two groups was observed for plasma retinol, which can be 
Table 3: Effect of stunting and place of living on plasma concentration of retinol, carotenoids and $\alpha$-tocopherol ( $\mu \mathrm{mol} / \mathrm{l}$ mean $\pm \mathrm{SD}$ ) of Laotian children

\begin{tabular}{lcccc}
\hline & Stunted & Non stunted & Lowland & Highland \\
\hline Girls / Boys & $25 / 15$ & $20 / 21$ & $25 / 18$ & $20 / 18$ \\
$\alpha$-Tocopherol & $1.701 \pm 1.818$ & $1.742 \pm 1.676$ & $1.965 \pm 1.796$ & $1.805 \pm 1.714$ \\
Retinol & $0.996 \pm 0.264$ & $0.937 \pm 0.223$ & $0.965 \pm 0.260$ & $0.969 \pm 0.118$ \\
Lutein & $0.055 \pm 0.051$ & $0.064 \pm 0.050$ & $0.062 \pm 0.049$ & $0.064 \pm 0.051$ \\
Zeaxanthin & $0.004 \pm 0.006$ & $0.003 \pm 0.002$ & $0.006 \pm 0.005$ & $0.003 \pm 0.002^{* * *}$ \\
$\beta$-Cryptoxanthin & $0.015 \pm 0.018$ & $0.011 \pm 0.017$ & $0.019 \pm 0.010$ & $0.011 \pm 0.018^{* * *}$ \\
$\alpha$-Carotene & $0.003 \pm 0.003$ & $0.006 \pm 0.009^{*}$ & $0.004 \pm 0.003$ & $0.006 \pm 0.009$ \\
$\beta$-Carotene & $0.011 \pm 0.019$ & $0.017 \pm 0.02 I^{*}$ & $0.017 \pm 0.020$ & $0.017 \pm 0.021$ \\
Lycopene & traces & traces & traces & traces \\
\hline
\end{tabular}

$* \mathrm{P}<0.05 * * * \mathrm{P}<0.001$

Table 4: Relative percentage of individual carotenoids in plasma of children from Europe and Laos (geometric mean - (geometric SD_U))

\begin{tabular}{|c|c|c|}
\hline & Europe & Laos \\
\hline $\mathrm{N}$ & 101 & 81 \\
\hline Lutein & $10.0(5.998)$ & $64.6(49.6) * * *$ \\
\hline Zeaxanthin & $0.6(0.4)$ & $0.3(0.0)$ \\
\hline$\beta$-Cryptoxanthin & $4.6(2.1)$ & $0.4(0.0) *$ \\
\hline$\alpha$-Carotene & $18.6(14.2)$ & $0.1(0.0)$ \\
\hline$\beta$-Carotene & $45.7(35.3)$ & $0.8(0.0)$ **** \\
\hline Lycopen & $11.5(5.2)$ & $0.0(0.0) *$ \\
\hline
\end{tabular}

$* \mathrm{P}<0.05 * * * \mathrm{P}<0.001$

explained by the homeostatic regulation of its plasma levels [26]. In none of the children plasma levels were indicative of deficiency. Despite low vitamin A intakes, plasma retinol concentrations of an average of $0.91-1.07 \mu \mathrm{mol} /$ 1 ( $95 \%$ C.I.) could be measured. Based on our protocol stunted children got $39 \%$ and non-stunted children got $43 \%$ of the recommended daily requirements. Other studies, however, show strong relationship between low vitamin A intake and low serum retinol concentrations [27]. The cause of this contradiction might be the homoeostatic regulation of the retinol and possible sufficient amounts present in the main storage organ for vitamin $\mathrm{A}$, the liver. As shown in Table 4 the effect of growth retardation and the area in which the children lived was of less importance for the level of retinol, carotenoids and $\alpha$ tocopherol in plasma.

Table 2 shows that in children from Laos substantial sex differences exist with regard to the levels of retinol, carotenoids and $\alpha$-tocopherol. In boys from Laos all components were significantly lower $(P<0.01)$. This is in contrast to our results from Europe and studies from Pakistan in children and from Algeria in adults, in which no such sex differences were observed $[13,22,24,28]$. In the study conducted in Pakistan however, levels of lycopene and $\beta$-cryptoxanthine were below detection limit and $\beta$ carotene was in traces in only $8 \%$ of the children and in the study from Algeria only $\beta$-carotene and $\alpha$-tocopherol were measured. The gender-specifically higher plasma concentrations of carotenoids in girls, however, have been reported by one study [29]. Another study found slightly higher levels of carotenoids only significant for lycopene in boys [23]. Based on the nutritional questionnaires taken in this study, this phenomenon cannot be explained by differences in nutrition. Additionally, the age of the children would exclude known effects of sex hormones on plasma levels of the investigated components.

\section{Conclusions}

Our results show that first, there is the possibility of using capillary blood plasma to evaluate retinol, carotenoids and $\alpha$-tocopherol as biomarkers of intake or status and the possible effect of diet on absolute and relative carotenoid composition. This modification including glass capillaries and a battery-driven centrifuge can substantially reduce the equipment necessary for plasma preparation, 
transportation and storage. Second, that the application of this method in Laotian children shows important factors that influence plasma levels of retinol, carotenoids and $\alpha$-tocopherol in Laotian infants such as sex and to a limited extent stunting and the region of origin.

\section{Competing interests}

None declared.

\section{Authors' contributions}

FJS participated in the conception, design, data analysis and the writing of the manuscript. JK participated in design, data analysis and writing of the manuscript. $\mathrm{AH}$ participated in the data analysis and the writing of parts of the manuscript. HJZ participated in the conception, design data analysis and critical revision of the manuscript. All authors have red and approved the last version of the manuscript.

\section{Acknowledgments}

The skilled assistance of all the participants of the Family Health Project in Laos and the support of the GTZ, Germany and Prof. Dr. Peter F. Schelp, Freie Universität zu Berlin, Germany is gratefully acknowledged.

\section{References}

I. Stephenson LS, Latham MC, Ottesen EA: Global malnutrition. Parasitology 2000, I 2 I (Suppl):S5-22.

2. Underwood BA, Arthur P: The contribution of vitamin A to public health. Faseb J 1996, 10(9): 1040-1048.

3. Stephensen $C B$ : Vitamin $A$, infection, and immune function. Annu Rev Nutr 200I, 21:167-192.

4. Thurnham DI: Micronutrients and immune function: some recent developments. J Clin Pathol I997, 50( I I):887-89 I.

5. Melikian G, Mmiro F, Ndugwa C, Perry R, Jackson JB, Garrett E, Tielsch J, Semba RD: Relation of vitamin A and carotenoid status to growth failure and mortality among Ugandan infants with human immunodeficiency virus. Nutrition 200I, 17(78):567-572.

6. van den Berg $\mathrm{H}$, van der Gaag M, Hendriks $\mathrm{H}$ : Influence of lifestyle on vitamin bioavailability. Int J Vitam Nutr Res 2002, 72(I):53-59.

7. Nestel $P$, Trumbo $P$ : The role of provitamin A carotenoids in the prevention and control of vitamin A deficiency. Arch Latinoam Nutr 1999, 49(3 Suppl I):26S-33S.

8. Sies $H$, Stahl $W$, Sundquist AR: Antioxidant functions of vitamins. Vitamins $E$ and $C$, beta-carotene, and other carotenoids. Ann N Y Acad Sci 1992, 669:7-20.

9. Russell RM: Physiological and clinical significance of carotenoids. Int J Vitam Nutr Res 1998, 68(6):349-353.

10. Rock E, Winklhofer-Roob BM, Ribalta J, Scotter M, Vasson MP, Brtko J, Brigelius-Flohe R, Bronner A, Azais-Braesco V: Vitamin A, vitamin $E$ and carotenoid status and metabolism during ageing: functional and nutritional consequences (VITAGE PROJECT). Nutr Metab Cardiovasc Dis 200 I, I I (4 Suppl):70-73.

II. El-Sohemy A, Baylin A, Kabagambe E, Ascherio A, Spiegelman D, Campos $\mathrm{H}$ : Individual carotenoid concentrations in adipose tissue and plasma as biomarkers of dietary intake. Am J Clin Nutr 2002, 76(1): 172-179.

12. Thurnham DI, Northrop-Clewes CA, Chopra M: Biomarkers of vegetable and fruit intakes. Am J Clin Nutr 1998, 68(3):756-758.

13. Abraham K, Grüters A, Wahn U, Müller C, Schweigert Fj: Minimal inflamation as study confounder for retinol, $\beta$-carotene as well as haematological and thyroidal parameters in children is revealed by an ultrasensitive C-reactive protein (CRP) assay. Int I Vitam Nutr Res 2003 in press.

14. Schweigert FJ, Steinhagen B, Raila J, Siemann A, Peet D, Buscher U: Conentrations of carotenoids, tocopherol and retinol in serum and follicular fluid of women undergoing IVF. Human Reprod 2003, I8: 1259-1264.
15. Thurnham DI, Northrop-Clewes CA: Optimal nutrition: vitamin A and the carotenoids. Proc Nutr Soc 1999, 58(2):449-457.

16. Thurnham DI: Vitamin A deficiency and its role in infection. Trans R Soc Trop Med Hyg 1989, 83(6):721-723.

17. Schweigert FJ: Inflammation-induced changes in the nutritional biomarkers serum retinol and carotenoids. Curr Opin Clin Nutr Metab Care 200I, 4(6):477-48I.

18. Thurnham DI, Singkamani R: The acute phase response and vitamin A status in malaria. Trans $R$ Soc Trop Med Hyg 1991, 85(2): 194-199.

19. Craft NE: Innovative approaches to vitamin A assessment. J Nutr 200I, I3I(5): 1626S-1630S.

20. Craft NE, Bulux J, Valdez C, Li Y, Solomons NW: Retinol concentrations in capillary dried blood spots from healthy volunteers: method validation. Am J Clin Nutr 2000, 72(2):450-454

2I. Erhardt JG, Heinrich F, Biesalski HK: Determination of retinol, antioxidant vitamins and homocysteine in skin puncture blood. Int J Vitam Nutr Res 1999, 69(5):309-3I4.

22. Lenhartz H, Schweitzer R, Botticher D, Leichsenring M: Plasma carotenoids in German children and adolescents. Eur J Pediatr 1997, I 56(I):71-73.

23. Ford ES, Gillespie C, Ballew C, Sowell A, Mannino DM: Serum carotenoid concentrations in US children and adolescents. $A m$ J Clin Nutr 2002, 76(4):818-827.

24. Thurnham DI, Northrop-Clewes CA, Paracha PI, McLoone UJ: The possible significance of parallel changes in plasma lutein and retinol in Pakistani infants during the summer season. $\mathrm{Br} J$ Nutr 1997, 78(5):775-784.

25. Becker K, Botticher D, Leichsenring M: Antioxidant vitamins in malnourished Nigerian children. Int J Vitam Nutr Res 1994, 64(4):306-310.

26. Blomhoff R: Transport and metabolism of vitamin A. Nutr Rev 1994, 52(2 Pt 2):SI3-23.

27. Zagre NM, Delisle H, Tarini A, Delpeuch F: [Changes in vitamin A intake following the social marketing of red palm oil among children and women in Burkina Faso]. Sante 2002, I 2(I):38-44.

28. Lachili B, Faure H, Arnaud J, Richard MJ, Benlatreche C, Favier A Roussel AM: Blood micronutrients in Algeria, relationships with sex and age. Int I Vitam Nutr Res 200 I, 7 I (2): I I I-II6.

29. Curran-Celentano J, Hammond BR Jr, Ciulla TA, Cooper DA, Pratt LM, Danis RB: Relation between dietary intake, serum concentrations, and retinal concentrations of lutein and zeaxanthin in adults in a Midwest population. Am J Clin Nutr 200I, 74(6):796-802.
Publish with Bio Med Central and every scientist can read your work free of charge

"BioMed Central will be the most significant development for disseminating the results of biomedical research in our lifetime. "

Sir Paul Nurse, Cancer Research UK

Your research papers will be:

- available free of charge to the entire biomedical community

- peer reviewed and published immediately upon acceptance

- cited in PubMed and archived on PubMed Central

- yours - you keep the copyright

Submit your manuscript here:

http://www.biomedcentral.com/info/publishing_adv.asp
BioMedcentral 